PROCEEDINGS OF THE

AMERICAN MATHEMATICAL SOCIETY

Volume 137, Number 2, February 2009, Pages 573-577

S 0002-9939(08)09574-9

Article electronically published on August 19, 2008

\title{
A NOTE ON ZEROES OF REAL POLYNOMIALS IN $C(K)$ SPACES
}

\author{
JESÚS FERRER \\ (Communicated by Nigel J. Kalton)
}

\begin{abstract}
For real $C(K)$ spaces, we show that being injected in a Hilbert space is a 3 -space property. As a consequence, we obtain that, when $K$ does not carry a strictly positive Radon measure, every quadratic continuous homogeneous real-valued polynomial on $C(K)$ admits a linear zero subspace enjoying a property which implies non-separability.
\end{abstract}

\section{TERMinOlogy AND PRELIMINARY RESUlts}

Throughout what follows $K$ will be a compact Hausdorff topological space and $C(K)$ its associated real-valued function space provided with the supremum norm. By $X$ and $X^{*}$ we denote a real Banach space and its topological dual, respectively, with the standard duality being represented by $\langle\cdot, \cdot\rangle$. If $A \subset X$ and $B \subset X^{*}$, we use the notation

$$
A^{\perp}=\left\{x^{*} \in X^{*}:\left\langle x^{*}, x\right\rangle=0, x \in A\right\}, \quad B_{\perp}=\left\{x \in X:\left\langle x^{*}, x\right\rangle=0, x^{*} \in B\right\} .
$$

Following the terminology introduced in [3, we say that a Banach space $X$ belongs to class $\mathcal{C}_{H}$ whenever $X$ is injected (i.e., there is a one-to-one bounded linear map) into a Hilbert space. Similarly, $\mathcal{W}^{*}$ is defined as the subclass of $\mathcal{C}_{H}$ formed by those spaces which are injected into a separable Hilbert space. The next few auxiliary results are quite straightforward and will be used in the sequel; see 3 for details.

Lemma 1. For a Banach space $X$ the following are equivalent:

(i) $X$ is in $\mathcal{W}^{*}$.

(ii) $X^{*}$ is weak*-separable.

(iii) $X^{*}$ contains a countable total subset.

(iv) There is a one-to-one bounded linear map from $X$ into a separable Banach space.

Lemma 2. If $Y$ is a closed linear subspace of $X$ such that $Y \in \mathcal{W}^{*}$ and $X / Y \in \mathcal{C}_{H}$, then $X \in \mathcal{C}_{H}$.

The following result is taken from [1, Corollary 3] and [6, Theorem 4.5].

Received by the editors January 23, 2008.

2000 Mathematics Subject Classification. Primary 47H60, 46B26.

Key words and phrases. Quadratic polynomials, zero-set, $C(K)$ spaces.

The author has been partially supported by MEC and FEDER Project MTM2005-08210.

(C)2008 American Mathematical Society 
Lemma 3. For a compact $K$, the following are equivalent:

(i) $C(K)$ belongs to $\mathcal{C}_{H}$.

(ii) $K$ carries a strictly positive Radon probability.

(iii) $C(K)^{*}$ contains a weakly compact total subset.

Although the property of being injected into a Hilbert space is not equivalent, in general, to that of being injected into a reflexive space (think of $\ell_{p}(\Gamma), \Gamma$ uncountable, $2<p<\infty)$, we see next that this equivalence is obtained for $C(K)$ spaces.

Proposition 1. For a compact $K, C(K)$ is in $\mathcal{C}_{H}$ if and only if it is injected into a reflexive space.

Proof. Since we only need to prove the sufficiency part, let $T: C(K) \rightarrow E$ be a one-to-one bounded linear map, where $E$ is a reflexive Banach space. Then, setting

$$
W:=T^{*}\left(B_{E^{*}}\right),
$$

where $B_{E^{*}}$ denotes the closed unit ball of the dual space $E^{*}$ and $T^{*}$ is the conjugate map of $T$, we have that $W$ is a weakly compact total subset of $C(K)^{*}$. Lemma 3 now applies.

Corollary 1. If $\Gamma$ is uncountable, then $c_{0}(\Gamma)$ is not injected into a reflexive space.

Proof. Since $c_{0}(\Gamma)$ is isomorphic to $C(K)$, where $K$ is taken to be the one-point compactification of the discrete space $\Gamma$, if $c_{0}(\Gamma)$ were injected into a reflexive space, we would have after the former proposition that $C(K) \in \mathcal{C}_{H}$. But, this would imply that $c_{0}(\Gamma) \in \mathcal{C}_{H}$, which is not so; see $[3$.

We say that a closed linear subspace $Y$ of $X$ is splitting whenever there is a bounded linear map $T: X \rightarrow Y$ such that $\operatorname{Ker} T \cap Y=\{0\}$. Notice that every complemented subspace of $X$ is splitting in $X$.

Proposition 2. Let $Y$ be a closed linear subspace which is splitting in $X$. If $Y$ and $X / Y$ are both injected into a reflexive space, then $X$ is also injected into a reflexive space.

Proof. Let $T: X \rightarrow Y, S_{1}: X / Y \rightarrow E_{1}, S_{2}: Y \rightarrow E_{2}$ be bounded linear maps, where $E_{1}$ and $E_{2}$ are reflexive spaces, such that $\operatorname{Ker} T \cap Y=\{0\}$ and $S_{1}, S_{2}$ are one-to-one. Defining $L: X \rightarrow E_{1} \times E_{2}$ as

$$
L x:=\left(S_{1}(x+Y), S_{2} T x\right), \quad x \in X,
$$

we obtain a bounded linear map into the reflexive space $E_{1} \times E_{2}$ such that it is also one-to-one: If $L x=(0,0)$, then we have that $S_{1}(x+Y)=0$ and $S_{2} T x=0$; hence $x \in Y \cap \operatorname{Ker} T=\{0\}$.

\section{A THREE-SPACE PROPERTY AND ZEROES OF QUADRATIC POLYNOMIALS ON $C(K)$}

In 2, Remark 3], the following question is posed: If $Y$ is a closed linear subspace of $X$ such that $Y$ and $X / Y$ are in class $\mathcal{C}_{H}$, does it necessarily follow that $X$ is also in this class? We show that the answer to this question is affirmative for $C(K)$ spaces. Later, we make use of this fact to see that continuous quadratic homogeneous polynomials on $C(K)$, with $K$ not carrying a strictly positive Radon measure, 
have a zero-set that contains linear subspaces with a property which implies nonseparability. This slightly improves Corollary 8 of [4, where an affirmative answer to the conjecture stated in [1, Remark 3] is given for $C(K)$ spaces. If $A$ is a subset of the compact $K$, by $C_{A}(K)$ we mean the closed linear subspace of $C(K)$ formed by those elements which vanish in $A$.

Proposition 3. Let $K_{0}$ be a closed subset of the compact space $K$ such that $C_{K_{0}}(K)$ is injected into a reflexive space. Then $C_{K_{0}}(K)$ is splitting in $C(K)$.

Proof. Since cozero sets, i.e., complements of the zero-sets of the elements of $C(K)$, form a base for the open sets of $K$, applying Zorn's Lemma, we can guarantee the existence of a maximal collection of pairwise disjoint cozero sets contained in the open set $K \backslash K_{0}$. This maximal collection has to be countable; otherwise there would be a copy of $c_{0}(\Gamma)$, with $\Gamma$ having the cardinality of the collection, contained in $C_{K_{0}}(K)$, but this would imply that $c_{0}(\Gamma)$ is injected into a reflexive space, a contradiction after Corollary 1. Thus, let $\left\{V_{j}: j \geq 1\right\}$ be this maximal collection. From the maximality and since $V:=\bigcup_{j=1}^{\infty} V_{j}$ is also a cozero set, there is $z \in C(K)$ such that

$$
V \subset K \backslash K_{0} \subset \bar{V}, \quad z^{-1}(0)=K \backslash V .
$$

Let $T$ be the map from $C(K)$ into $C_{K_{0}}(K)$ defined as

$$
T x:=x \cdot z, \quad x \in C(K) \text {. }
$$

Then, $T$ is well defined, linear and bounded. Besides, if $x \in \operatorname{Ker} T \cap C_{K_{0}}(K)$, it follows that $x_{\mid K_{0}}=0$ and $x z=0$; consequently, since $z(t) \neq 0, t \in V$, we have that $x_{\mid \bar{V}}=0$ and so $x=0$.

Proposition 4. Let $Y$ be a closed linear subspace of $C(K)$ such that $C(K) / Y$ is injected into a reflexive space. Then, there is a Radon probability $\mu$ on $K$ such that $C_{\text {supp } \mu}(K)$ is contained in $Y$.

Proof. Let $T: C(K) / Y \rightarrow E$ be a one-to-one bounded linear map, with $E$ reflexive. Let $F$ be the closure of $T^{*}\left(E^{*}\right)$ in $(C(K) / Y)^{*}=Y^{\perp}$. Since $F$ is weakly compactly generated, no copy of $\ell_{1}(\Gamma), \Gamma$ uncountable, can be contained in $F$; thus, after [6, Lemma 1.3], we have that there is a Radon probability $\mu$ on $K$ such that every element of $F$ is $\mu$-absolutely continuous. Hence, for each $u^{*} \in E^{*}$, there is a $\mu$-measurable function $f_{u^{*}}$ which is the Radon-Nikodym derivative of $T^{*} u^{*}$ with respect to $\mu$ and so

$$
\left\langle T^{*} u^{*}, x\right\rangle=\int_{K} x d\left(T^{*} u^{*}\right)=\int_{K} x f_{u^{*}} d \mu, \quad x \in C(K) .
$$

Thus, if $K_{0}:=\operatorname{supp} \mu$, we have that, for $x \in C_{K_{0}}(K)$,

$$
\left\langle T^{*} u^{*}, x\right\rangle=\int_{K_{0}} x f_{u^{*}} d \mu=0
$$

that is

$$
C_{K_{0}}(K) \subset \bigcap_{u^{*} \in E^{*}} \operatorname{Ker} T^{*} u^{*}
$$

But $T^{*}$ has weak ${ }^{*}$-dense range in $Y^{\perp}$. Therefore

$$
Y^{\perp}={\overline{T^{*}\left(E^{*}\right)}}^{w^{*}}=\left(\bigcap_{u^{*} \in E^{*}} \operatorname{Ker} T^{*} u^{*}\right)^{\perp},
$$

which leads to $C_{K_{0}}(K) \subset Y$. 
Corollary 2. If $Y$ is a closed linear subspace of $C(K)$ such that $Y$ and $C(K) / Y$ are injected into a reflexive space, then $C(K)$ is in $\mathcal{C}_{H}$.

Proof. From Proposition 4, we have that there is a closed subset $K_{0}$ of $K$ supporting a Radon probability such that $C_{K_{0}}(K) \subset Y$. Hence, we have that $C_{K_{0}}(K)$ is injected into a reflexive space and, from Proposition 3 , that $C_{K_{0}}(K)$ is splitting in $C(K)$. After Lemma 3, since $C(K) / C_{K_{0}}(K)$ is isomorphic to $C\left(K_{0}\right)$, we obtain that $C(K) / C_{K_{0}}(K)$ is in $\mathcal{C}_{H}$. The result now follows from Proposition 2 and Proposition 1.

Corollary 3. If $Y$ is a closed linear subspace of $C(K)$ such that $Y$ and $C(K) / Y$ are in $\mathcal{C}_{H}$, then $C(K)$ is also in $\mathcal{C}_{H}$; i.e., being injected into a Hilbert space is a 3 -space property for $C(K)$ spaces.

Concerning this 3-space property, this author has learned from the editor, Professor Nigel Kalton, that the problem of whether being injected into a Hilbert space is a 3 -space property has a negative solution in general. The particular counterexample to this problem (also provided by Professor Kalton) is the following:

Let $\Gamma$ be an uncountable set and consider the Banach space $Z_{2}(\Gamma)$, defined similarly to $Z_{2}(\mathbb{N})$ in [5, Section 6]. For our purposes, it suffices to recall the following properties of $Z_{2}(\Gamma)$ : It is a reflexive space which can be normed in such a way that it has a closed linear subspace $Y$ isometric to $\ell_{2}(\Gamma)$ with $Z_{2}(\Gamma) / Y$ isometric to $\ell_{2}(\Gamma)$; i.e., $Z_{2}(\Gamma)$ is a twisted sum of $\ell_{2}(\Gamma)$ with itself. Furthermore, $Z_{2}(\Gamma)^{*}$ is isometric to $Z_{2}(\Gamma)$. Thus, $Y$ and $Z_{2}(\Gamma) / Y$ are in class $\mathcal{C}_{H}$, while we show that $Z_{2}(\Gamma) \notin \mathcal{C}_{H}$ : Let $T: Z_{2}(\Gamma) \rightarrow H$ be any bounded linear map, where $H$ is a Hilbert space. After [5, Theorem 6.5, Corollary 6.8], it can be deduced that every such map is strictly singular, so the restriction of $T$ to the subspace $\ell_{2}(\Gamma)$ is therefore compact and is not one-to-one. Consequently, $T$ cannot be one-to-one either.

In [1, Remark 3], the following dichotomy is conjectured: For a real Banach space $X$, either it admits a positive definite continuous quadratic homogeneous polynomial or every continuous quadratic homogeneous polynomial has a zero-set that contains a non-separable linear subspace. In [4, using a weaker form of the 3-space property obtained before, the above conjecture is seen to be correct for $C(K)$ spaces. Our next result is a slight improvement of this.

Let us just recall that if $P$ is a continuous quadratic homogeneous polynomial in $X$, then $P^{\prime}: X \rightarrow X^{*}$ represents the Fréchet derivative map, i.e., a bounded linear map such that

$$
\left\langle P^{\prime}(x), y\right\rangle=2 \stackrel{\vee}{P}(x, y), \quad x, y \in X,
$$

where $\stackrel{\vee}{P}$ stands for the symmetric bilinear functional associated to $P$.

Corollary 4. If $C(K) \notin \mathcal{C}_{H}$, then for each continuous quadratic homogeneous real-valued polynomial $P$ in $C(K)$, every maximal linear subspace $Z$ contained in $P^{-1}(0)$ satisfies that either $Z \notin \mathcal{W}^{*}$ or $\overline{P^{\prime}(Z)}$ is not weakly compactly generated.

Proof. Zorn's Lemma guarantees the existence of maximal linear subspaces contained in the polynomial's zero-set $P^{-1}(0)$. Let $Z$ be one of such maximal linear subspaces, which is clearly closed. Seeking a contradiction, let us assume that $Z \in \mathcal{W}^{*}$ and $\overline{P^{\prime}(Z)}$ is weakly compactly generated. Again using Rosenthal's dichotomic result of [6, Lemma 1.3], we know that there is a Radon probability $\mu$ on 
$K$ such that every element of $\overline{P^{\prime}(Z)}$ is $\mu$-absolutely continuous. Let $Y:=P^{\prime}(Z)_{\perp}$ and $K_{0}:=\operatorname{supp} \mu$. Proceeding as in the proof of Proposition 4 , it is easy to see that $C_{K_{0}}(K)$ is contained in $Y$. Besides, $Z \subset Y$ and the maximality of $Z$ yield that $P^{-1}(0) \cap Y=Z$, and so the polynomial $P$ does not change sign in $Y$; thus, defining $\widetilde{P}(x+Z):=P(x), x \in Y$, we have a continuous quadratic polynomial $\widetilde{P}$ on $Y / Z$ which is positive (or negative) definite. This implies that $Y / Z \in \mathcal{C}_{H}$; see 1. Proposition 2]. Hence, after Lemma 2, it follows that $Y \in \mathcal{C}_{H}$. Then, we have that $C_{K_{0}}(K) \in \mathcal{C}_{H}$ and since $C(K) / C_{K_{0}}(K)$ is isomorphic to $C\left(K_{0}\right)$,

$$
C(K) / C_{K_{0}}(K) \in \mathcal{C}_{H} .
$$

The desired contradiction follows from Corollary 3.

Notice that if $P$ is a continuous quadratic homogeneous real-valued polynomial on the Banach space $X$, then the linear map $P^{\prime}$ given by the Fréchet derivative satisfies that $\operatorname{Ker} P^{\prime} \subset P^{-1}(0)$. Thus, our next result gives us a quite large linear subspace contained in the polynomial's zero-set for a wide class of compacta.

Corollary 5. If $C(K) \notin \mathcal{C}_{H}$ and $C(K)^{*} \in \mathcal{C}_{H}$, then for each continuous quadratic homogeneous polynomial $P, \operatorname{Ker} P^{\prime} \notin \mathcal{C}_{H}$.

Proof. Since $C(K) / \operatorname{Ker} P^{\prime}$ is injected into $C(K)^{*}$, it follows that $C(K) / \operatorname{Ker} P^{\prime} \in$ $\mathcal{C}_{H}$. Hence, from Corollary 3 the result is obtained.

If $K$ is a scattered compact with an uncountable amount of isolated points, then $C(K) \notin \mathcal{C}_{H}$, while $C(K)^{*}=\ell_{1}(K) \in \mathcal{C}_{H}$. Hence, after the previous corollary, the next result follows.

Corollary 6. Let $K$ be a scattered compact space with uncountably many isolated points. Then, for each quadratic continuous homogeneous polynomial $P, \operatorname{Ker} P^{\prime} \notin$ $\mathcal{C}_{H}$.

\section{ACKNOWLEDGEMENTS}

The author is grateful to the referee for a number of suggestions which have certainly improved the paper and also to Professor Nigel Kalton, who has kindly provided some information containing the definite negative solution to the 3 -space problem of being injected into a Hilbert space.

\section{REFERENCES}

[1] R.M. Aron, C. Boyd, R.A. Ryan, I. Zalduendo, Zeros of polynomials on Banach spaces: The real story, Positivity 7 (2003), pp. 285-295. MR2017308 (2004j:46066)

[2] T. Banakh, A. Plichko, A. Zagorodnyuk, Zeros of quadratic functionals on non-separable spaces, Colloq. Math. 100, No. 1 (2004), pp. 141-147. MR2079354 (2005g:46047)

[3] J. Ferrer, On the zero-set of real polynomials in non-separable Banach spaces, Publ. of R.I.M.S. 43, No. 3 (2007), pp. 685-697. MR2361792

[4] J. Ferrer, Zeroes of real polynomials on $C(K)$ spaces, J. Math. Anal. Appl. 336 (2007), pp. 788796. MR 2352980

[5] N. J. Kalton, N. T. Peck, Twisted sums of sequence spaces and the three-space problem, Trans. Amer. Math. Soc. 255 (1979), pp. 1-30. MR542869 (82g:46021)

[6] H. P. Rosenthal, On injective Banach spaces and the spaces $L^{\infty}(\mu)$ for finite measures $\mu$, Acta Math. 124 (1970), pp. 205-248. MR0257721(41:2370)

Departamento de Análisis Matemático, Universidad de Valencia, Dr. Moliner, 50, 46100 Burjasot (VAlencia), Spain

E-mail address: Jesus.Ferrer@uv.es 